\title{
The GraRS regulatory system controls Staphylococcus aureus susceptibility to antimicrobial host defenses
}

\author{
Dirk Kraus $^{1}$, Silvia Herbert ${ }^{2}$, Sascha A Kristian ${ }^{3,4}$, Arya Khosravi ${ }^{3}$, \\ Victor Nizet ${ }^{3}$, Friedrich Götz ${ }^{2}$ and Andreas Peschel*1
}

\begin{abstract}
Address: ${ }^{1}$ Cellular and Molecular Microbiology Division, Institute for Medical Microbiology and Hygiene, University of Tübingen, ElfriedeAulhorn-Str. 6, 72076 Tübingen, Germany, ${ }^{2}$ Microbial Genetics, University of Tübingen, Tübingen, Germany, ${ }^{3}$ Departments of Pediatrics, University of California, San Diego, La Jolla, California, USA and ${ }^{4}$ Veterans Affairs San Diego Healthcare System, La Jolla, California, USA

Email: Dirk Kraus - dirk.kraus@med.uni-tuebingen.de; Silvia Herbert - silvia.herbert@uni-tuebingen.de; Sascha A Kristian - skristia@ucsd.edu; Arya Khosravi - akhosrav@usc.edu; Victor Nizet - vnizet@ucsd.edu; Friedrich Götz - friedrich.goetz@uni-tuebingen.de; Andreas Peschel* - andreas.peschel@med.uni-tuebingen.de

* Corresponding author
\end{abstract}

Published: 2 June 2008

BMC Microbiology 2008, 8:85 doi:10.1 |86/147|-2/80-8-85
Received: 18 January 2008

Accepted: 2 June 2008

This article is available from: http://www.biomedcentral.com/I47I-2/80/8/85

(C) 2008 Kraus et al; licensee BioMed Central Ltd.

This is an Open Access article distributed under the terms of the Creative Commons Attribution License (http://creativecommons.org/licenses/by/2.0), which permits unrestricted use, distribution, and reproduction in any medium, provided the original work is properly cited.

\begin{abstract}
Background: Modification of teichoic acids with D-alanine by the products of the dit operon protects Gram-positive bacteria against major antimicrobial host defense molecules such as defensins, cathelicidins, myeloperoxidase or phospholipase. The graRS regulatory genes have recently been implicated in the control of D-alanylation in Staphylococcus aureus.

Results: To determine the impact of the GraRS regulatory system on resistance to antimicrobial host defense mechanisms and virulence of $S$. aureus, we compared inactivation of S. aureus SAII 3 wild type and its isogenic graRS deletion mutant by the human cathelicidin LL-37 or human neutrophil granulocytes in vitro, and the ability to cause infection in vivo. We show here that graRS deletion considerably alters bacterial surface charge, increases susceptibility to killing by human neutrophils or the defense peptide LL-37, and attenuates virulence of $S$. aureus in a mouse infection model.
\end{abstract}

Conclusion: Our results indicate that $S$. aureus can regulate its surface properties in order to overcome innate host defenses.

\section{Background}

Staphylococcus aureus, a frequent cause of human infections, is highly resistant to antimicrobial factors of the innate immune system such as cationic antimicrobial peptides (CAMPs) $[1,2]$ which are produced by epithelial cells and neutrophils $[3,4]$. These peptides generally contain 10-50 amino acids and have positive net charges [5]. Due to their cationic properties, CAMPs can easily bind to the highly negatively charged bacterial cell envelope and inactivate bacteria, e.g. by forming pores in the bacterial membrane leading to bacterial lysis [6]. S. aureus has evolved mechanisms to alter the anionic charge of cell surface components to gain resistance to a broad variety of cationic antimicrobial factors such as CAMPs [7], phospholipase A2 [8], myeloperoxidase [9], or lysozyme [10]. One such mechanism is based upon modification of phospholipids in the cytoplasmic membrane by introducing a positively charged lysyl group into anionic phosphatidylglycerol by the MprF protein, thereby neutralizing the net charge of the membrane surface 
$[11,12]$. A similar reaction is mediated by products of the dltABCD operon, which are responsible for attachment of positively charged D-alanine residues into negatively charged phosphate groups in the backbone of teichoic acids $[7,9]$. Mechanisms involved in the regulation of these resistance factors are not yet well understood in Gram-positive bacteria. Herbert et al. recently found that the $S$. aureus two-component regulatory system graRS positively regulates expression of the dlt operon. In a S. aureus SA113 graRS deletion mutant, the content of D-alanine in teichoic acids was reduced by $47 \%$ and the mutant showed reduced resistance to various antibiotics including polymyxin $\mathrm{B}$, gallidermin, and vancomycin $[10,13]$. Accordingly, graRS have previously been implicated in regulation of vancomycin intermediary resistance [14]. As the dlt operon plays a key role in $S$. aureus resistance to cationic antimicrobial host molecules, the graRS system may be important in evasion of host defense mechanisms such as cationic antimicrobial peptides and neutrophil killing.

\section{Results}

The graRS mutant shows altered cell surface charge but unaltered lysyl-phosphatidylglycerol (LPG) content

In order to study if reduced expression of the dlt operon upon graRS disruption results in altered cell surface charge, we compared binding of the red-coloured, cationic protein cytochrome $c$ to wild type $S$. aureus SA113 (WT), the isogenic graRS mutant, and the plasmid-complemented mutant.

The graRS mutant bound significantly more cytochrome $c$ than the WT or the complemented mutant (Fig. 1A), which is in accordance with the recently described reduced content of D-alanine residues in the teichoic acids of the mutant [10].

To analyse whether increased binding of cytochrome $c$ by the graRS mutant is in fact due to altered cell surface charge by decreased teichoic acid alanylation or the altered expression of other surface-exposed molecules, we also examined binding of cytochrome $c$ to a dltA deletion mutant, which completely lacks D-alanine substitution of teichoic acids [7], and a dltA/graRS double deletion mutant, which was generated by transducing the graRS mutation into the dltA mutant. Due to the high binding capacities of the dltA and the dltA/graRS mutant we modified the conditions in order to prevent limitation of applied cytochrome $c$. Deletion of the graRS genes in the dlt mutant background did not lead to further increased binding of cytochrome $c$ compared to the dltA mutant (Fig. 1B). This finding indicates that the increased binding of cytochrome $c$ by the graRS mutant is due to decreased alanylation of teichoic acids and that the resulting surface charge alteration does not result from other regulatory effects mediated by GraRS.
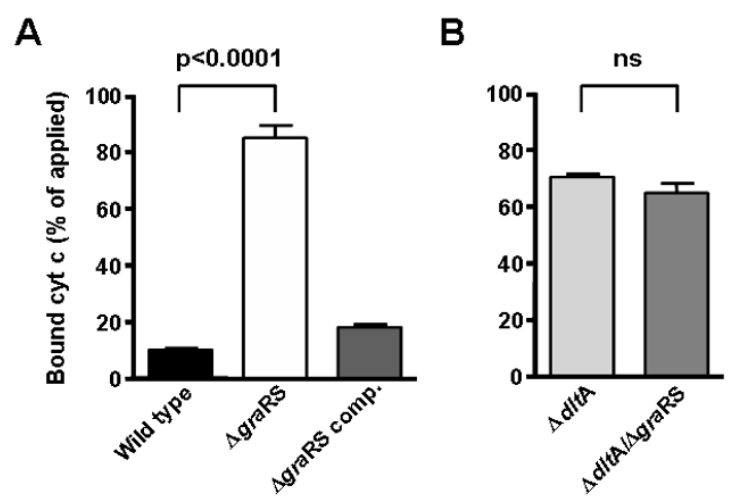

\section{Figure I}

Binding of cationic cytochrome c (cyt c) by S. aureus Sa I 3 wild type, graRS mutant, and complemented graRS mutant (A) and by the ditA mutant and the dltA/graRS double mutant (B). Bacteria were incubated with $500 \mu \mathrm{l}$ cytochrome $c$ solution $(0.5 \mathrm{mg} / \mathrm{ml})(\mathrm{A})$ or $750 \mu \mathrm{l}$ cytochrome $c$ solution $(\mathrm{I} \mathrm{mg} / \mathrm{ml})(B)$. The means and SEM of at least three independent experiments are shown. $P$ values $<$ 0.05 as calculated by Student's $t$ test were regarded statistically significant.

Increased binding of cationic proteins may also result from reduced $m p r F$ expression and, accordingly, reduced lysylphosphatidylglycerol (LPG) content. To control for this possibility, we compared patterns of membrane lipids from log-phase bacteria by thin-layer chromatography. The amounts of LPG from WT and graRS mutant were indistinguishable (data not shown), which corroborates recent findings that $m p r F$ is not among the graRS-regulated genes in S. aureus SA113 [10].

The graRS mutant is more susceptible to killing by LL-37 and human neutrophil granulocytes in vitro

In an attempt to test whether the increased affinity of the graRS mutant to cationic molecules leads to higher susceptibility to human host defense peptides, we compared inactivation of WT and graRS mutant by the human cathelicidin LL-37. This antimicrobial peptide is active against $S$. aureus at high concentrations or long exposure [15] but in this experiment we chose conditions under which the LL-37 did not affect viability of the S. aureus WT. Accordingly, the WT and complemented mutant strains showed no significant decrease in CFU following LL-37 exposure, whereas the number of graRS mutant bacteria recovered was only $25 \%$ of the original inoculum (Fig. 2 A).

Next we investigated whether the graRS mutant is killed faster than the parental strain by human neutrophils, which produce high amounts of LL-37 and other CAMPs as components of their antibacterial killing arsenal. The 
A

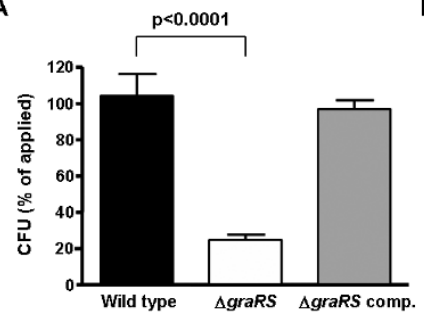

B

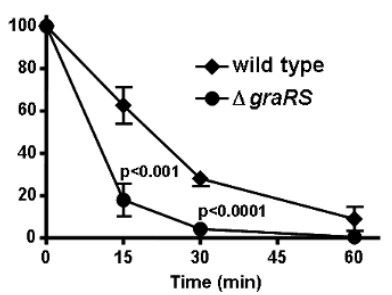

Figure 2

(A) Inactivation of wild type (black bar), graRS mutant (white bar) and complemented mutant (grey bar) by LL-37 after 20-min incubation with $20 \mu \mathrm{g} / \mathrm{ml}$ LL-37. (B) Inactivation of wild-type (squares) and mutant bacteria (circles) by human neutrophils after I5, 30 and 60 minutes. The means and SEM of three independent experiments run in duplicate $(A)$ and the means and SD of two independent experiments run in duplicate (B) are shown. $P$ values $<0.05$ as calculated by Student's $t$ test were regarded statistically significant.

graRS mutant was killed by neutrophils considerably faster than the WT strain. After 15 and $30 \mathrm{~min}$, the recovered CFU of the graRS mutant were significantly lower than those of the WT (Fig 2B). Of note, our previous studies had shown that altered alanylation of teichoic acids does not affect the rate of PMN phagocytosis [9]. Taken together, these data indicate that graRS-mediated control of CAMP resistance mechanisms is of importance for $S$. aureus evasion from neutrophil killing.

Deletion of graRS leads to attenuated virulence in a mouse infection model

To investigate the impact of reduced resistance of the graRS mutant to neutrophil and CAMP-mediated killing on the ability of the bacteria to cause infections in vivo, we compared the virulence of WT and mutant bacteria in a mouse challenge model. Therefore female BALB/c mice (12 to 15 weeks old) were infected with $S$. aureus WT or graRS mutant bacteria. $72 \mathrm{~h}$ after infection numbers of $\mathrm{CFU} /$ kidney were determined.

Significantly less bacteria were detected in the kidneys of animals, which had been infected with the graRS mutant than those infected with the WT bacteria. (Fig. 3) This finding is in coincidence with the increased susceptibility to clearance by CAMPs and neutrophils, corroborating the central importance of these host factors in innate defense.

\section{Discussion}

A large variety of regulatory systems has been described in $S$. aureus during the last decades [16-18]. While many systems have been shown to control adhesion and toxin production [19], much less is known about the regulation of

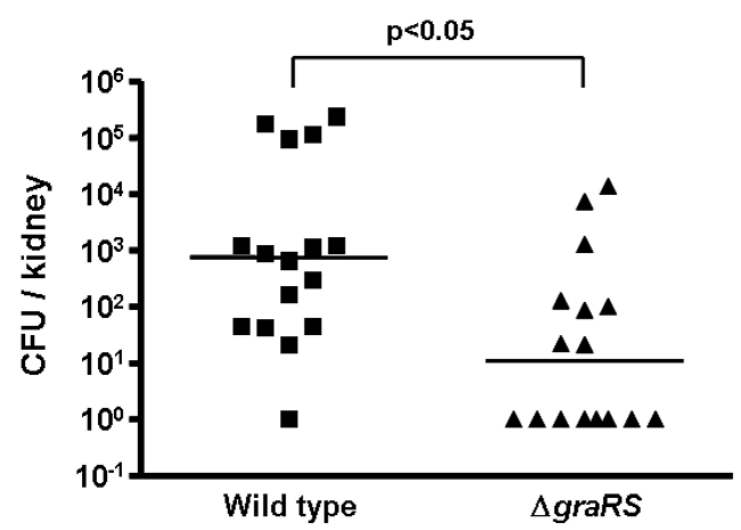

Figure 3

CFU of wild-type and mutant bacteria in kidneys of mice $72 \mathrm{~h}$ after intraperitoneal infection with $1.2 \times$ $10^{8}$ bacteria. For each strain 16 animals were tested. Medians are given as horizontal lines. A $P$ value $<0.05$ as calculated by Student's test was regarded statistically significant.

genes involved in the resistance to antimicrobial peptides, such as the dlt genes. However, the critical role of this operon in infection $[7,9]$ suggests that it may be appropriately regulated in response to environmental stimuli. Listeria monocytogenes expresses the VirR transcription factor, which regulates both the dlt operon and the mprF gene [20]. The S. aureus regulatory genes rot and arl have previously been shown to have a moderate influence on transcription of the dlt operon $[21,22]$. We have recently demonstrated that inactivation of the GraRS system leads to only $13 \%$ of the wild-type transcription level of the $d l t$ operon and a decreased level of teichoic acid alanylation in S. aureus SA113 [10]. Other important genes regulated by graRS in $S$. aureus include the vraFG genes involved in the resistance to vancomycin $[10,13]$. Similar data have recently been presented for the Staphylococcus epidermidis aps system [23]. This three component regulatory system is homologous to the graRS system of $S$. aureus. However, when initially describing the graRS system in S. aureus, Meehl et al. failed to recognize the three-component nature of this system [13]. Li et al. recently showed that deletion of graS in the community-associated MRSA strain MW2 leads to decreased minimal inhibitory concentrations of some cationic antimicrobial peptides such as the human beta-defensin hBD3 or the human cathelicidin LL37 and, similar to our studies, to decreased virulence of the mutant in a mouse infection model [24]. Furthermore, they could show that several important factors in CAMP resistance including the dlt operon and $m p r F$ are activated by a diverse panel of antimicrobial peptides [24]. The fact that $m p r F$ is not regulated by graRS in $S$. aureus SA113 could be due to the deficiency of this strain in the global gene regulatory system agr [25] or to other genetic defects. 


\section{Conclusion}

We could show here that, besides its role in influencing the effectiveness of pharmacologic antimicrobials, the GraRS regulatory system plays a key role in resistance to natural antimicrobials of our innate immune system against $S$. aureus and merits further attention in an era of increasing reports of virulent and drug-resistant strains of this foremost human pathogen.

\section{Methods \\ Strains and growth conditions}

Staphylococcus aureus SA113 (WT), the isogenic graRS deletion mutant [10], the plasmid-complemented graRS deletion mutant [10], the isogenic dltA deletion mutant [7] and the isogenic $d l t \mathrm{~A} / \mathrm{graRS}$ double deletion mutant were inoculated in basic medium (BM; $1 \%$ tryptone, $0.5 \%$ yeast extract, $0.5 \% \mathrm{NaCl}, 0.1 \%$ glucose, $0.1 \% \mathrm{~K}_{2} \mathrm{HPO}_{4}$ ) with aliquots of overnight cultures and incubated at $37^{\circ} \mathrm{C}$ until logarithmic phase was reached. In case of the complemented mutant, BM was modified by replacing glucose with $0.5 \%$ xylose to allow for expression of the plasmidencoded graRS genes [10].

The graRS::erm/dltA::spc double mutant was created by bacteriophage $\phi$ 11-mediated transduction of the graRS::erm mutation into the dltA::spc deletion mutant.

To prepare bacteria for the mouse infection model, precultures of the staphylococcal strains which were grown for 8 $\mathrm{h}$ in tryptic soy broth (TSB) were diluted 1:100 into fresh TSB and incubated for $18 \mathrm{~h}$ without shaking.

\section{Cytochrome c binding assay}

Log-phase bacteria were harvested, washed twice with potassium phosphate buffer containing $0.01 \%$ human serum albumin (KPi buffer) and bacterial density was adjusted to an $\mathrm{OD}_{600}$ of 3 . Bacteria from $1.5 \mathrm{ml}$ aliquots were resuspended in $500 \mu \mathrm{l}$ cytochrome $c$ (Sigma) solution $(0.5 \mathrm{mg} / \mathrm{ml})$ in case of the wild type, the graRS mutant and the complemented graRS mutant and $750 \mu \mathrm{l}$ cytochrome $c$ (Sigma) solution $(1 \mathrm{mg} / \mathrm{ml})$ in case of the $d l t \mathrm{~A}$ and the $d l t \mathrm{~A} / \mathrm{graRS}$ double mutant, respectively, and incubated at $37^{\circ} \mathrm{C}$. In order to prevent bacterial sedimentation, samples were vigorously shaken. After $15 \mathrm{~min}$, samples were centrifuged and the supernatant was assayed photometrically at $410 \mathrm{~nm}$.

\section{Lipid analyses}

Comparison of membrane lipid patterns by extraction of polar lipids and subsequent thin-layer chromatography was performed as recently described [11].

\section{Inactivation assay with human defense peptide LL-37}

Log-phase bacteria were harvested, washed twice with potassium phosphate buffer containing 0.01\% human serum albumin (KPi buffer) and bacterial density was adjusted to an $\mathrm{OD}_{600}$ of 1.5. Samples $(40 \mu \mathrm{l})$ with a final concentration of $20 \mu \mathrm{g} / \mathrm{ml} \mathrm{LL}-37$ were shaken at $37^{\circ} \mathrm{C}$. After $20 \mathrm{~min}, 160 \mu \mathrm{l}$ ice-cold KPi buffer was added to block further antimicrobial action and appropriate aliquots were plated on BM agar plates. After $24 \mathrm{~h}$ incubation at $37^{\circ} \mathrm{C}$, CFU were enumerated.

\section{Inactivation assay with human neutrophil granulocytes}

Bacteria were grown to logarithmic phase, washed, and adjusted in KPi buffer as described above. Neutrophils were isolated from peripheral blood of healthy volunteers by ficoll/histopaque gradient centrifugation as described previously [26] and resuspended in HBSS-HSA (HBSS containing $0.05 \%$ human serum albumin). Bacterial and neutrophil suspensions were mixed to final concentrations of $5 \times 10^{6} / \mathrm{ml}$ bacteria and $2.5 \times 10^{6} / \mathrm{ml}$ neutrophils. Bacteria were opsonized by addition of pooled human serum (Sigma) to a final concentration of $10 \%$. Samples $(500 \mu \mathrm{l})$ were shaken at $37^{\circ} \mathrm{C}$. After 15,30 and $60 \mathrm{~min}$, aliquots were diluted in ice-cold water and vortexed vigorously to disrupt the neutrophils and halt bacterial killing. Appropriate dilutions were plated on BM agar plates and incubated at $37^{\circ} \mathrm{C}$ for $24 \mathrm{~h}$ for enumeration of CFU.

\section{Mouse infection model}

All procedures involving animals were approved by the UCSD Animal Care Committee, which serves to ensure that all federal guidelines concerning animal experimentation are met.

Female BALB/c mice (12 to 15 weeks old) were infected intraperitoneally with $S$. aureus WT or the graRS mutant. Briefly, precultures of the staphylococcal strains which were grown for $8 \mathrm{~h}$ in tryptic soy broth (TSB) were diluted 1:100 into fresh TSB, incubated for $18 \mathrm{~h}$ without shaking, washed twice in PBS, adjusted to $3 \times 10^{8} \mathrm{CFU} / \mathrm{ml}$ in PBS and $400 \mu \mathrm{l}$ of these suspensions were injected intraperitoneally. $72 \mathrm{~h}$ after infection, mice were sacrificed, one kidney was aseptically removed, weighed, homogenized, and serially diluted in PBS for plating on Todd Hewitt agar plates. After $24 \mathrm{~h}$ incubation at $37^{\circ} \mathrm{C}$, the numbers of $\mathrm{CFU} /$ kidney were determined.

\section{Authors' contributions}

DK did all experiments except for the mouse infection model, which was done by SAK and AK. AP supervised research and wrote the paper. SH generated the graRS/dltA double mutant. AP, FG and VN conceived the study and analyzed results. All authors read and approved the final manuscript.

\section{Acknowledgements}

We thank Gabriele Hornig for excellent technical assistance, Hubert Kalbacher for LL-37 and Nadine Göhring for critically reading the manuscript. Our research is supported by grants from the German Research Founda- 
tion (TR34, FOR449, GRK685, SFB766), the European Union (LSHM-CT2004-5 I 2093), the German Ministry of Education and Research (NGFN2), the IZKF program of the Medical Faculty, University of Tübingen, to AP.

\section{References}

I. Foster TJ: Immune evasion by staphylococci. Nat Rev Microbiol 2005, 3:948-958.

2. Peschel A, Sahl HG: The co-evolution of host cationic antimicrobial peptides and microbial resistance. Nat Rev Microbiol 2006, 4:529-536

3. Jenssen H, Hamill P, Hancock RE: Peptide antimicrobial agents. Clin Microbiol Rev 2006, 19:49I-5II.

4. Lehrer RI, Ganz T: Defensins of vertebrate animals. Curr Opin Immunol 2002, 14:96-102.

5. Hancock RE, Sahl HG: Antimicrobial and host-defense peptides as new anti-infective therapeutic strategies. Nat Biotechnol 2006, 24: I55I-I557.

6. Sahl HG, Pag U, Bonness S, Wagner S, Antcheva N, Tossi A: Mammalian defensins: structures and mechanism of antibiotic activity. J Leukoc Biol 2005, 77:466-475.

7. Peschel A, Otto M, Jack RW, Kalbacher H, Jung G, Götz F: Inactivation of the dlt operon in Staphylococcus aureus confers sensitivity to defensins, protegrins, and other antimicrobial peptides. J Biol Chem 1999, 274:8405-84I0.

8. Koprivnjak T, Peschel A, Gelb MH, Liang NS, Weiss JP: Role of charge properties of bacterial envelope in bactericidal action of human group IIA phospholipase A2 against Staphylococcus aureus. J Biol Chem 2002, 277:47636-47644.

9. Collins LV, Kristian SA, Weidenmaier C, Faigle M, van Kessel KP, van Strijp JA, Götz F, Neumeister B, Peschel A: Staphylococcus aureus strains lacking $D$-alanine modifications of teichoic acids are highly susceptible to human neutrophil killing and are virulence attenuated in mice. $J$ Infect Dis 2002, 186:2I4-219.

10. Herbert S, Bera A, Nerz C, Kraus D, Peschel A, Goerke C, Meehl M, Cheung A, Götz F: Molecular basis of resistance to muramidase and cationic antimicrobial peptide activity of lysozyme in staphylococci. PLoS Pathog 2007, 3:e 102.

II. Peschel A, Jack RW, Otto M, Collins LV, Staubitz P, Nicholson G, Kalbacher $H$, Nieuwenhuizen WF, Jung G, Tarkowski A, van Kessel KP, van Strijp JA: Staphylococcus aureus resistance to human defensins and evasion of neutrophil killing via the novel virulence factor MprF is based on modification of membrane lipids with I-lysine. J Exp Med 200I, 1 93:1067-1076.

12. Staubitz P, Neumann H, Schneider T, Wiedemann I, Peschel A: MprFmediated biosynthesis of lysylphosphatidylglycerol, an important determinant in staphylococcal defensin resistance. FEMS Microbiol Lett 2004, 23 I:67-7I.

13. Meehl M, Herbert S, Götz F, Cheung A: Interaction of the GraRS Two-Component System with the VraFG ABC-Transporter to Support Vancomycin-Intermediate Resistance in Staphylococcus aureus. Antimicrob Agents Chemother 2007.

14. Cui L, Lian JQ, Neoh HM, Reyes E, Hiramatsu K: DNA microarraybased identification of genes associated with glycopeptide resistance in Staphylococcus aureus. Antimicrob Agents Chemother 2005, 49:3404-3413.

15. Weidenmaier C, Kokai-Kun JF, Kristian SA, Chanturiya T, Kalbacher H, Gross M, Nicholson G, Neumeister B, Mond JJ, Peschel A: Role of teichoic acids in Staphylococcus aureus nasal colonization, a major risk factor in nosocomial infections. Nat Med 2004, 10:243-245.

16. Bronner S, Monteil H, Prevost G: Regulation of virulence determinants in Staphylococcus aureus: complexity and applications. FEMS Microbiol Rev 2004, 28:183-200.

17. Cheung AL, Bayer AS, Zhang G, Gresham H, Xiong YQ: Regulation of virulence determinants in vitro and in vivo in Staphylococcus aureus. FEMS Immunol Med Microbiol 2004, 40:I-9.

18. Novick RP: Autoinduction and signal transduction in the regulation of staphylococcal virulence. Mol Microbiol 2003, 48:1429-1449.

19. Ziebandt AK, Becher D, Ohlsen K, Hacker J, Hecker M, Engelmann S: The influence of agr and sigmaB in growth phase dependent regulation of virulence factors in Staphylococcus aureus. Proteomics 2004, 4:3034-3047.

20. Mandin P, Fsihi H, Dussurget O, Vergassola M, Milohanic E, ToledoArana A, Lasa I, Johansson J, Cossart P: VirR, a response regulator critical for Listeria monocytogenes virulence. Mol Microbiol 2005, 57:1367-1380.

21. Koprivnjak T, Mlakar V, Swanson L, Fournier B, Peschel A, Weiss JP: Cation-induced transcriptional regulation of the dlt operon of Staphylococcus aureus. J Bacteriol 2006, I88:3622-3630.

22. Said-Salim B, Dunman PM, McAleese FM, Macapagal D, Murphy E, McNamara PJ, Arvidson S, Foster TJ, Projan SJ, Kreiswirth BN: Global regulation of Staphylococcus aureus genes by Rot. J Bacteriol 2003, 185:610-619.

23. Li M, Lai Y, Villaruz AE, Cha DJ, Sturdevant DE, Otto M: Gram-positive three-component antimicrobial peptide-sensing system. Proc Natl Acad Sci U S A 2007, 104:9469-9474.

24. Li M, Cha DJ, Lai Y, Villaruz AE, Sturdevant DE, Otto M: The antimicrobial peptide-sensing system aps of Staphylococcus aureus. Mol Microbiol 2007, 66: I I 36- I I 47.

25. Vuong C, Saenz HL, Götz F, Otto M: Impact of the agr quorumsensing system on adherence to polystyrene in Staphylococcus aureus. J Infect Dis 2000, 182:1688-1693.

26. Schmitz FJ, Veldkamp KE, van Kessel KP, Verhoef J, van Strijp JA: Delta-toxin from Staphylococcus aureus as a costimulator of human neutrophil oxidative burst. J Infect Dis 1997, 176:1531-1537.
Publish with Bio Med Central and every scientist can read your work free of charge

"BioMed Central will be the most significant development for disseminating the results of biomedical research in our lifetime. "

Sir Paul Nurse, Cancer Research UK

Your research papers will be:

- available free of charge to the entire biomedical community

- peer reviewed and published immediately upon acceptance

- cited in PubMed and archived on PubMed Central

- yours - you keep the copyright

Submit your manuscript here:

http://www.biomedcentral.com/info/publishing_adv.asp
BioMedcentral 